\title{
Role of chemotherapy for treatment of colorectal liver metastasis in modern era of liver surgery. Current data and clinical practice in 2017
}

\author{
Iswanto Sucandy* and Darrell J Downs \\ Digestive Disease Institute, Florida Hospital Tampa, USA
}

Surgical resection is the only potentially curative treatment for colorectal liver metastasis (CLM) with reported 5-year survival rates of $30-40 \%$ [1]. At least $50 \%$ of all patients with colorectal cancer develop liver metastasis during their lifetime. Despite negative margins during liver resection, recurrent disease in the liver remains common. In the 1970's when liver resections were historically performed without use of chemotherapy, the five-year overall survival after complete tumor resection was less than $20 \%$ [2]. Perioperative chemotherapy, mainly in the adjuvant setting, has then been employed as a method to decrease tumor recurrence and increase overall survival post liver resection. Because promising outcomes were seen with the use of chemotherapy in patients with unresectable or borderline resectable liver metastases, concept of neoadjuvant chemotherapy for resectable colorectal liver metastasis has gained popularity among hepatobiliary surgeons and medical oncologists. However, the role of neoadjuvant chemotherapy for resectable colorectal liver metastasis is still controversial. Only limited data is available on the literature regarding this issue. We aimed to summarize current clinical evidence of utilizing neoadjuvant chemotherapy for patients with resectable CLM.

In the original Intergroup trial report published by Nordlinger et al. which compared outcomes of perioperative chemotherapy and liver resection versus liver resection alone, only $63 \%$ of patients in chemotherapy group actually received the adjuvant chemotherapy. Of those who received adjuvant chemotherapy, only $70 \%$ completed their treatment [3]. A significant number of patients did not receive or complete their chemotherapy due to postoperative complications, intolerable toxic effects of chemotherapy, disease progression while on chemotherapy, and patient refusal. However, potential advantages of administering neoadjuvant chemotherapy in resectable cases are early treatment of micrometastatic disease, reduction of tumor volume/size (which often translates to optimum liver parenchymal preservation), and selection (subsequent exclusion from liver resection) of patients with rapidly progressing tumor which indicates aggressive tumor biology.

A systematic review of all currently available evidence since year 2000 was performed. All English literatures, which described comparative outcomes of liver resection for colorectal metastasis with and without neoadjuvant chemotherapy were reviewed. Studies containing patients with unresectable metastatic disease or borderline resectable disease were excluded since the use of chemotherapy in these patients had been uniformly accepted to be beneficial. Only one randomized clinical trial study has been published regarding the use of perioperative chemotherapy for resectable colorectal liver metastasis. However, this study by Nordlinger et al was not specifically designed to differentiate clinical outcomes between neoadjuvant and adjuvant chemotherapy in combination with surgical resections ${ }^{3}$. Patients who received neoadjuvant chemotherapy prior to their liver resections were found to have an increased 3-year progression free survival. Overall survival, however, was not affected by administration of neoadjuvant chemotherapy. A follow-up study five years later by the same group failed to show prolongation of progression-free and overall survival in patients who received neoadjuvant chemotherapy prior to liver resections. Other studies are retrospective in nature with highly heterogenous reported data points. A careful analysis must be employed during interpretation, since many of the published reports have obvious selection biases. Patients with more than two hepatic tumors and/or unfavorable tumor locations, larger/borderline resectable, bilateral lesions, short disease free interval, and higher clinical risk score tend to be started on neoadjuvant chemotherapy to evaluate their invivo response, prior to liver resection. Those with single lesion located peripherally were scheduled for immediate liver resections followed by postoperative chemotherapy. Previously published studies that reported overall 3- and 5-year survival outcomes in neoadjuvant group and non-neaodjuvant group are summarized in Table 1 [3-10]. Only a single study which was published by Tanaka et al in 2003 demonstrated significant 5 -year overall survival benefit in patients who received neoadjuvant chemotherapy for resectable colorectal liver metastasis (38.9\% versus $20.7 \%, \mathrm{p}=0.039$ ) [4].

Despite potential advantages of neoadjuvant chemotherapy for resectable liver lesions, this method of administration also comes with potential disadvantages. Liver damages in the form of hepatic steatosis/ steatohepatitis secondary to irinotecan and sinusoidal obstruction (hepatic veno-occlusive disease) secondary to oxaliplatin are well known complications. These chemotherapy regiments induce parenchymal changes that could increase postoperative morbidity. Approximately $7-10 \%$ of patients who undergo neoadjuvant chemotherapy progress to unresectable disease, which exclude them from a potentially curative surgical treatment. Furthermore, neoadjuvant chemotherapy may also lead to disappearance of small metastases on radiological imaging, which could result in technically more difficult curative operation.

Correspondence to: Iswanto Sucandy, Digestive Disease Institute, Florida Hospital Tampa, 3000 Medical Park Dr, Suite \#500, Tampa, Florida 33613, USA; Tel: (813) 615-7030; E-mail: iswanto_sucandy@yahoo.com

Key words: Chemotherapy, Colorectal Cancer, Liver Metastasis

Received: September 10, 2017; Accepted: September 26, 2017; Published: September 29, 2017 
Table 1. Survival outcomes in studies reporting effects of neoadjuvant chemotherapy in patients with resectable colorectal liver metastasis

\begin{tabular}{|c|c|c|c|c|c|c|c|}
\hline Study & Author & Year & Neoadjuvant Group & Surgery Group & Neoadjuvant Group & Surgery Group & P-Value \\
\hline & & & 3 year OS & 3 year OS & 5 year OS & 5 year OS & \\
\hline 1 & Tanaka et al & 2003 & $67 \%$ & $51.80 \%$ & $38.90 \%$ & $20.70 \%$ & 0.039 \\
\hline 2 & Scoggins et al & 2009 & $\mathrm{nr}$ & $\mathrm{nr}$ & $\mathrm{nr}$ & $\mathrm{nr}$ & ns \\
\hline 3 & Boostrom et al & 2009 & $62 \%$ & $63 \%$ & $48 \%$ & $45 \%$ & ns \\
\hline 4 & Pinto et al & 2012 & $59 \%$ & $71 \%$ & $43 \%$ & $55 \%$ & ns \\
\hline 5 & Oh et al & 2012 & $44 \%$ & $67 \%$ & $\mathrm{nr}$ & $\mathrm{nr}$ & ns \\
\hline 6 & Nordlinger et al & 2013 & $\mathrm{nr}$ & $\mathrm{nr}$ & $51.20 \%$ & $47.80 \%$ & ns \\
\hline 7 & Araujo et al & 2013 & $78 \%$ & $74 \%$ & $74 \%$ & $56 \%$ & ns \\
\hline 8 & Zhu et al & 2014 & $69 \%$ & $\mathrm{nr}$ & $52 \%$ & $48 \%$ & ns \\
\hline \multicolumn{8}{|c|}{$\begin{array}{l}\mathrm{ns}=\text { no significance } \\
\mathrm{OS}=\text { overall survival } \\
\mathrm{nr}=\text { details not reported }\end{array}$} \\
\hline
\end{tabular}

Notably, administration of neoadjuvant chemotherapy was associated with a trend toward higher rate of postoperative complications $(37.2 \%$ versus $24 \%, \mathrm{p}=0.006$ ). Therefore, age, comorbidities, and patient characteristics should all be taken into consideration, in order to optimize the usefulness of preoperative chemotherapy.

Thus far, neoadjuvant chemotherapy was found to improve 3-year progression free survival in only one prospective randomized study. While postoperative chemotherapy has been the standard of care for colorectal liver metastasis, data on the usefulness of neoadjuvant chemotherapy in resectable lesions is still currently nonconclusive. Neoadjuvant chemotherapy has been widely administered in the United States by community medical oncologists for resectable colorectal liver metastasis, even before referring the patient to a liver surgeon or a multidisciplinary discussion. There has been no consensus or guideline in regard to the most effective combination of drugs and schedule of administration. Different chemotherapeutic protocols are used in different institutions. Folfox and folfiri have been widely administered, but combinations of oxalipatin, fluoropyrimidine, leucovorin, capecitabine, irinotecan, and monoclonal antibodies are also commonly used in many hepatobiliary centers (both community and academic). Consequently, response rates to the neoadjuvant regiments are variable (ranging from $33 \%$ to $61 \%$ ) with disease progression rates ranging from $7 \%$ to $47 \%$ [5]. In conclusions, neoadjuvant chemotherapy is currently the standard of care for borderline resectable liver metastasis from colorectal cancer. In cases of resectable lesions, however, there have been no convincing prospective randomized clinical trial which shows superiority when compared to straight liver resection, even though neoadjuvant chemotherapy is given widely in clinical practice for patients with resectable lesions. Prospective randomized trial would be important to objectively determine benefits of neoadjuvant chemotherapy in resectable liver metastasis from colorectal cancer.

\section{References}

1. Nigri G, Petrucciani N, Ferla F, La Torre M, Aurello P, et al. (2015) Neoadjuvant chemotherapy for resectable colorectal liver metastases: what is the evidence? Results of a systematic review of comparative studies. Surgeon 13: 83-90. [Crossref]

2. Adson MA (1979) Hepatic metastasis. Hepatic resection. Dis Colon Rectum 22: 366369. [Crossref]

3. Nordlinger B, Sorbye H, Glimelius B, Poston GJ, Schlag PM, et al. (2008) Perioperative chemotherapy with FOLFOX4 and surgery versus surgery alone for resectable liver metastases from colorectal cancer (EORTC Intergroup trial 40983): a randomised controlled trial. Lancet 22: 1007-1016. [Crossref]

4. Tanaka K, Adam R, Shimada H, Azoulay D, Lévi F, et al. (2003) Role of neoadjuvant chemotherapy in the treatment of multiple colorectal metastases to the liver. Br J Surg 90: 963-969. [Crossref]

5. Scoggins CR, Campbell ML, Landry CS, Slomiany BA, Woodall CE, et al. (2009) Preoperative chemotherapy does not increase morbidity or mortality of hepatic resection for colorectal cancer metastases. Ann Surg Oncol 16: 35-41. [Crossref]

6. Boostrom SY, Nagorney DM, Donohue JH, Harmsen S, Thomsen K, et al. (2009) Impact of neoadjuvant chemotherapy with FOLFOX/FOLFIRI on disease-free and overall survival of patients with colorectal metastases. J Gastrointest Surg 13: 20032009. [Crossref]

7. Oh SY, Kim DY, Kim YB, Suh KW (2013) Comparison of oncological outcomes between neoadjuvant and adjuvant chemotherapy combined with surgery for resectable synchronous colorectal liver metastases. J Surg Res 182: 257-263. [Crossref]

8. Araujo R, Gonen M, Allen P, Blumgart L, DeMatteo R, et al. (2013) Comparison between perioperative and postoperative chemotherapy after potentially curative hepatic resection for metastatic colorectal cancer. Ann Surg Oncol 20: 4312-4321. [Crossref]

9. Zhu D, Zhong Y, Wei Y, Ye L, Lin Q, et al. (2014) Effect of neoadjuvant chemotherapy in patients with resectable colorectal liver metastases. PLoS One 9: e86543. [Crossref]

10. Pinto Marques H, Barroso E, de Jong MC, Choti MA, Ribeiro V, et al. (2012) Perioperative chemotherapy for resectable colorectal liver metastasis: does timing of systemic therapy matter? J Surg Oncol 105: 511-519. [Crossref]

Copyright: (C2017 Sucandy I. This is an open-access article distributed under the terms of the Creative Commons Attribution License, which permits unrestricted use, distribution, and reproduction in any medium, provided the original author and source are credited. 